\title{
Aplicación de la evaluación On-line en asignaturas de contenido morfológico
}

\author{
Isabel López Cortés ${ }^{\mathrm{a}^{*}}$, Domingo Carlos Salazar García ${ }^{\mathrm{b}}$, Borja \\ Velázquez Martí \\ a) Departamento de Producción Vegetal. Universidad Politécnica de Valencia. \\ Camino de Vera s /n. 46022 Valencia (Spain). islocor@upv.es \\ ${ }^{b)}$ Departament de Prehistòria i Arqueologia, Universitat de València. Av. Blasco \\ Ibáñez 28, 46010 Valencia, España.Domingo_carlos@ \\ c) Departamento de Ingeniería Rural y Agroalimentaria. Universidad Politécnica de \\ Valencia. Camino de Vera s/n. 46022 Valencia (Spain).borvemar@upv.es
}

\begin{abstract}
The use of active participatory methodologies and therefore the students, practical content attribute meanings and encourage student participation. Allows commitment to the construction of implicating knowledge in the use of materials of initiation provided and designed by teachers but students independently, both individually and collectively, will be expanded and completing then move to a forum such documents and comments tutorial for feedback and participation are drawn directly.

This material if necessary be completed, but this possible expansion will be made after public exposure in the classroom and to all colleagues. So the work is evaluated by the collective student-teachers (at least two) or possibly by titrating On-line contributions made by students. Later this documentation after the collaborative contributions of the entire group of students, will be uploaded to the platform online resources. This documentary will be generated based on the specific topic in view of the ongoing assessment of students and if the basis for global or recuperative assessment for certain students in the group material.

Therefore, the action will create database documentation by the teacher, submit it to work for a particular student who sent the group (group of students and teacher), which collaboratively expanded to subsequently submit it to a wider forum the platform.

Thus we get feedback that will lead to efficient participatory documentation, which is the objective sought in this work. This documentation generated will serve for continuous and personalized assessment of the performers, but also be the basis for the overall assessment of the subject or the subject for nonparticipating students.
\end{abstract}

Keywords: active methodologies, e-learning, continuous assessment, on-line teaching. 


\section{Resumen}

El uso de metodologías activas y por tanto participativas del alumnado, atribuyen significados prácticos a los contenidos y fomentan la participación del alumno. Permite el compromiso en la construcción del conocimiento implicandosé en el uso de materiales de iniciación aportados y diseñados por el profesorado pero que el alumno de forma autónoma, tanto individual como colectivamente, irá ampliando y completando para posteriormente pasar a un foro aquellos documentos y comentarios que por retroalimentación y con participación tutorial se van elaborando.

Este material si es necesario se completará, pero esta posible ampliación será realizada, tras la exposición pública en aula y ante todos los compañeros. De forma que el trabajo es evaluado por el colectivo alumnos-profesores (en un mínimo de dos) o en su caso mediante valoración On-line de las aportaciones realizadas por los alumnos. Posteriormente esta documentación tras las aportaciones colaborativas de todo el grupo de alumnos, será subido a la plataforma de recursos on line. Ello será la base documental generada sobre el tema concreto con vistas a las evaluaciones continuas del alumnado y en su caso el material base para la evaluación global o recuperativa para determinados alumnos del grupo.

Por lo tanto, la acción será crear una documentación base por parte del profesor, someterla a trabajo de un alumno concreto que enviará al colectivo (conjunto de alumnos y profesor), el cual de forma colaborativa ampliarán para posteriormente someterla a un foro más amplio en la plataforma.

De esta forma conseguiremos una retroalimentación que conducirá a una documentación participativa eficiente, que es el objetivo buscado en este trabajo. Esta documentación generada servirá para la evaluación continua y personalizada de los actuantes, pero también será la base para la evaluación global de la temática o de la asignatura para el alumnado no participativo.

Palabras clave: metodologías activas, e-learning, evaluación continua, didáctica On-line. 


\section{Introducción}

Cada vez es más claro que las metodologías didácticas han cambiado y están cambiando hoy con técnicas metodológicas activas, se busca la máxima implicación de los alumnos en las tareas docentes, participando en las actividades de laboratorio y campo sino también en aula de docencia teórica.

La participación activa no es posible sin niveles muy altos de motivación en el alumnado, así como unos objetivos claros, previamente con el alumnado, que les conduzca a tener y tomar iniciativas participativas no solo en la construcción de los programas sino también es necesaria su participación, no como una actividad puntual y sin continuidad, sino como forma clara de construir su propio conocimiento en base a unos datos previos aportados por el profesorado y sobre los que el alumnado trabajará completándolos (Biscarri, 2006).

Conocer materiales, ya sean vegetales que es el ejemplo que vamos a tomar, como constructivos en el pasado en arqueología y el presente en construcción, su caracterización y utilidad más o menos adecuada, al menos por lo que a usos y aplicaciones concretos se les conocen son básicas en el comienzo de cualquier asignatura.

El conocimiento del material sobre el que se trabaja en un área que tradicionalmente ha generado confusión en el alumnado es esencial. Por ello, pretendemos generar unas técnicas actuacionales que estimulen actitudes y que motiven al alumnado, fomenten su participación y contribuyan al mejor desarrollo de su capacidad. De esta forma implementaremos usos y alternativas a estos materiales vegetales situándolos en lugares y épocas reales, pero sin perder la capacidad buscar o incluso intuir los usos y aplicabilidad de estos y otros materiales, así como profundizar en tecnologías para su elaboración y empleo.

Atribuir significado práctico a los conocimientos es actualmente esencial en el proceso educativo.

Olvidar el concepto "yo enseño y tú memorizas" es importante o "yo hablo y tú escuchas" ambos son principios a olvidar en la pedagogía moderna (Luisel y Rodríguez, 2014). Es importante que el alumno trabaje sobre lo que ya conoce o sobre unos documentos aportados por el profesor y que el alumno efectúe sus aportaciones y experiencia lo que es imprescindible en las nuevas técnicas educativas que incorporan participación, creatividad y poder de síntesis. Si en el pasado se habló de la creatividad del profesor hoy se debe hablar de la creatividad del binomio alumno-profesor y es en este contexto donde se incluyen el aprendizaje significativo que implica un proceso constructivo activo y continuo del conocimiento (Díaz, 1999).

Recordemos que muchas de las competencias que queremos que posean nuestros alumnos, solo se adquieren cuando son ejercitadas. Y el ejercicio en la elaboración de fichas que permitan el conocimiento de las materias primeras de cualquier tipo es esencial para a partir de ellas y tras la caracterización de las mismas, desarrollar los procesos tecnológicos que conduzcan a su aplicación o consumo racional y razonado.

(c) ) EY-Nc-ND 2016, Universitat Politècnica de València

Congreso In-Red (2016) 


\section{Desarrollo de la innovación}

Ausubel ya en 2002 habla de tres tipos de aprendizaje el de preposiciones (de secuencias expositivas), el de conceptos y el de representaciones, nosotros en este caso hemos insistido en el de aprendizaje de representaciones mediante diagramas que pretendemos mejorar durante el desarrollo de la tarea.

Se presenta al alumnado una ficha base descriptiva y un mecanismo de trabajo como es el uso de códigos de identificación como, para el caso de material vegetal pueden ser normas de la Unión para la Protección de las Obtenciones Vegetales (UPOV) y su actualización para España, normas Community lant Variety Office (CPVO), normas de calidad o normas del proyecto de Recursos Genéticos (RESGEN), así como documentos ejemplo.

Se comienza con un aprendizaje autónomo consistente en la búsqueda de diferentes fichas varietales, en este caso del material en estudio, parte de la bibliografía se les incorpora en soporte digital a través de la plataforma PolitormaT, otra parte está a su disposición en el aula laboratorio y otra parte debe ser localizada mediante los buscadores habituales en estas temáticas (Marcelo, 2007).

Se suministra al alumno unas fotografías que permiten conocer básicamente el material, este material estará además a su disposición en el aula laboratorio, siempre que sea de temporada, o conservado en colección en caso contrario. Siempre debemos excepto en temas arqueológicos evitar el concepto de museo o colección estática. 


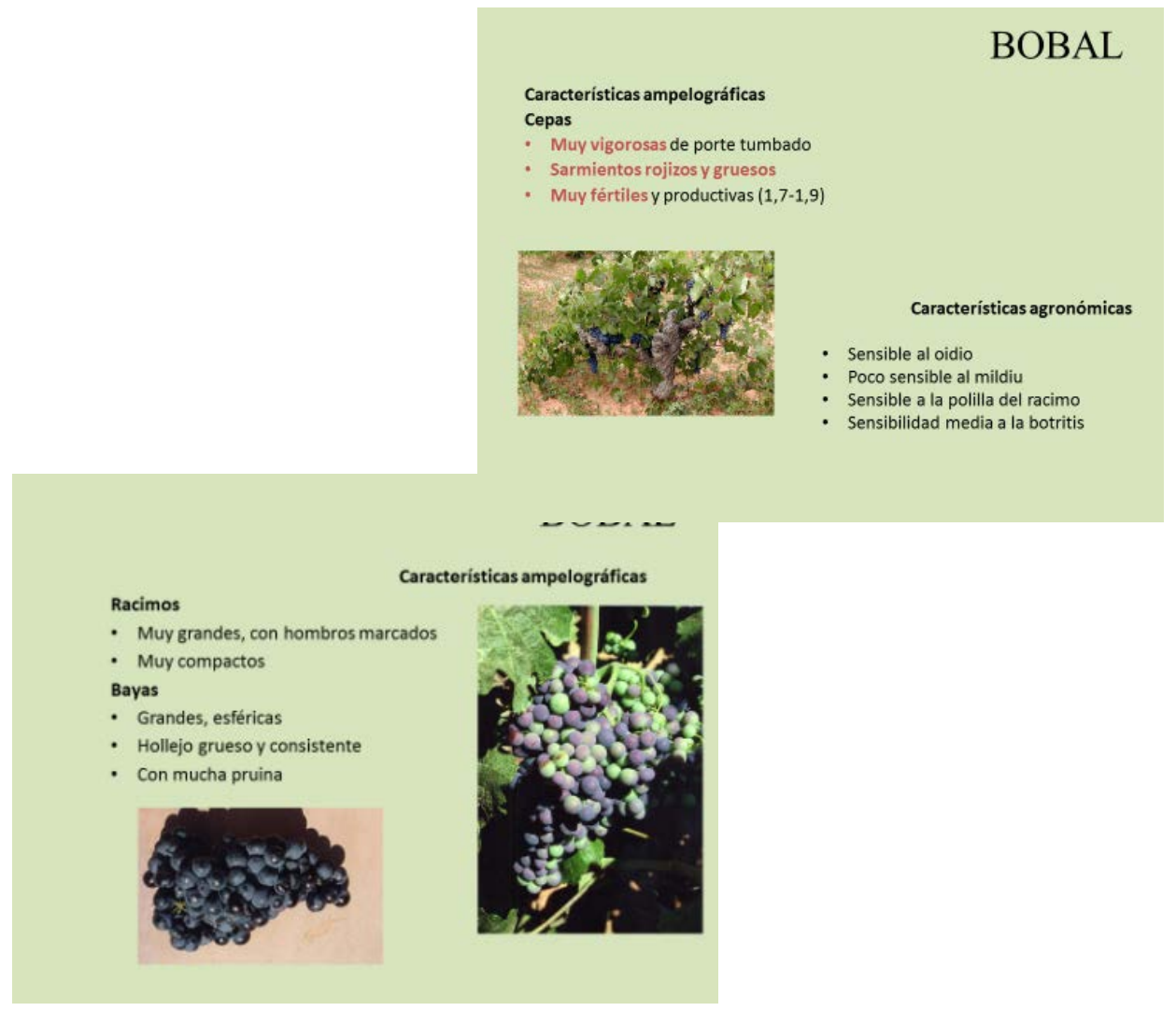

Fig. 1.- Modelo de ficha resumida elaborada por el profesorado (fase primera).

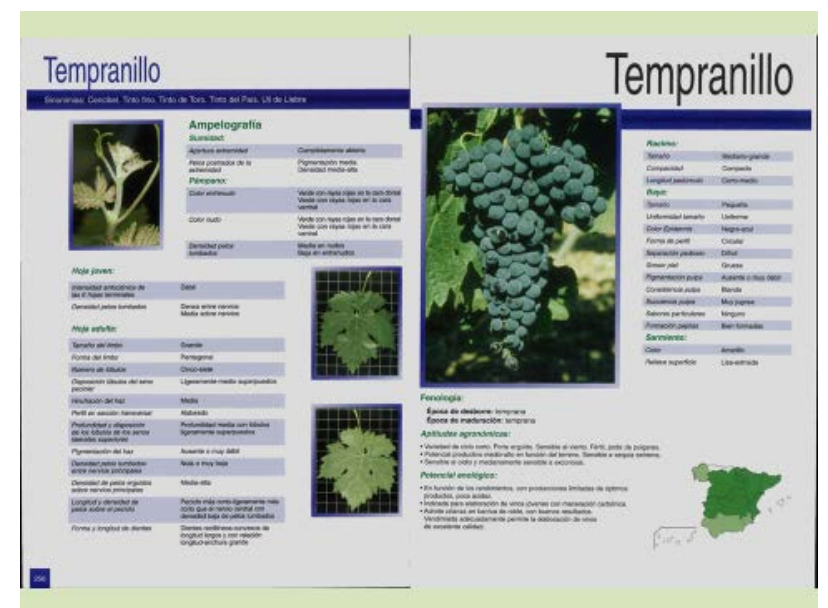

Fig. 2.- Modelo de ficha estructurada presentada por el profesor (fase primera).

(cc) BY-NC-ND 2016, Universitat Politècnica de València

Congreso In-Red (2016) 
Tras la búsqueda de otros materiales documentales, gráficos o físicos sobre el producto a estudiar se plantearán técnicas de aprendizaje cooperativo que pueden comenzar con la búsqueda de estos materiales en concreto, lo que es muy fácil y un estímulo claro en el caso de la búsqueda de uva de un cultivar concreto y unos vinos procedentes de la variedad en estudio, que es el ejemplo primero que vamos a emplear en el caso que desarrollamos.

Esta metodología descrita tiene un inconveniente, la necesidad de una dedicación fuera del aula, en la búsqueda en establecimientos del material, pero que al ser una actividad cooperativa aunque la inicie un alumno como tarea propia puede realmente ser efectuada no solo bibliográficamente, lo que es necesario, sino también con determinadas actividades, aplicaciones, degustaciones, catas, visionado de documentales, etc.

Posteriormente se preparan unos cuadros para que los alumnos primero de forma personalizada y luego en colectividad vayan elaborando la ficha que se desea obtener de cada uno de los materiales que sean propuestos ya sean vegetales o restos antropológicos.

El foro de este trabajo de aprendizaje que debe ser claramente interactivo ha sido ensayado por nuestro grupo en tres asignaturas, sumamente diversas, de la manera que en ellas se ha actuado se indica a continuación:

El profesorado propone el material sobre el que se va a trabajar y de donde procede según De Candolle (1813), Negroul (1946) y Vavilov (1987) y en el caso que nos ocupa en este ejemplo sobre material vegetal.

El alumno responsable de la elaboración de la ficha del material vegetal, recogiendo la ficha básica aportada por el profesor, expone sus resultados mediante una presentación a todos los alumnos de la asignatura que aportarán detalles complementarios en la elaboración activa de la ficha que se van incorporando en el mismo momento si es posible, una vez el alumno responsable ha expuesto y adicionado su presentación se establece un diálogo en el aula que se valora por el número de cuestiones planteadas y por las aportaciones.

Finalmente la ficha elaborada y ya con las aportaciones efectuadas por el colectivo serán incorporadas al foro creado y se contempla la retroalimentación y evaluación de este acto concreto con un pequeño cuestionario a contestar en un periodo corto de tiempo.

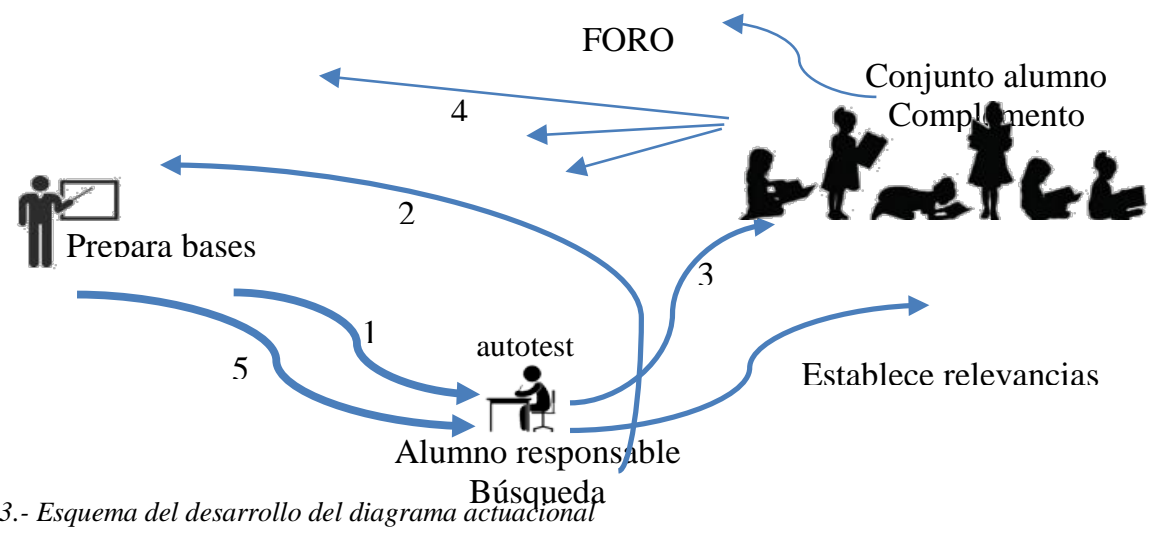

(cc) EY-NC-ND 2016, Universitat Politècnica de València 


\section{Resultados}

Los resultados obtenidos son clasificados en función de las evaluaciones realizadas, de esta forma se decidió, conjuntamente con el alumnado, establecer tres evaluaciones como se indica a continuación:

primera evaluación.

Temática: presentación ficha descriptiva básica

Sistema evaluación: Participativa, es evaluada por cada alumno y por los profesores

Resultados: 100\% evaluación positiva

segunda evaluación.

Temática: Aportación por alumnos en grupo

Sistema evaluación: Por al menos dos profesores y autoevaluación del alumnado

Resultados: Como se observa en figura 4a la valoración de esta evaluación se reparte en cuestiones planteadas por los alumnos y aportes a la ficha en elaboración.

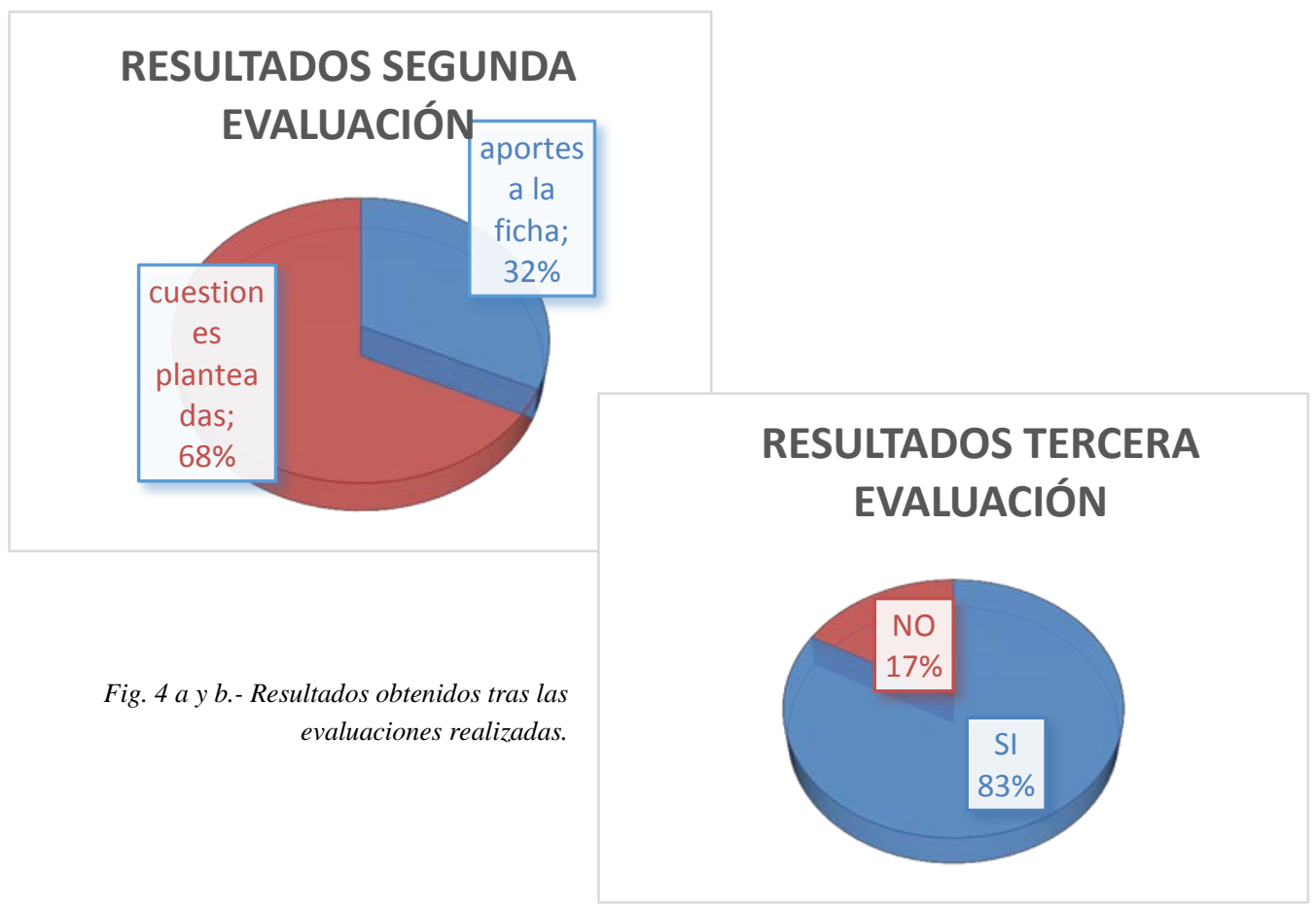

(cc) EY-Nc-No 2016, Universitat Politècnica de València

Congreso In-Red (2016) 


\section{Tercera evaluación.}

Temática: Participación en foros

Sistema evaluación: Automática y corregida por el alumno responsable

Resultados: Como se observa en el figura $4 \mathrm{~b}$ es mayoritariamente afirmativa

La contestación recibida en los primeros tres días es potenciada mediante puntos adicionales en la evaluación, de esta forma conseguimos mejorar las acciones realizadas por el alumnado. Ha podido observarse en los últimos años que para algunos grupos de alumnos es habitual, cuando no es respuesta inmediata, olvidar la participación en alguna actividad, como se observa en la Tabla 1 hemos conseguido invertir esta tendencia marcando unos plazos para las contestaciones.

\begin{tabular}{cccccc}
\hline $\begin{array}{c}\mathbf{N}^{\circ} \text { de } \\
\text { alumnos }\end{array}$ & $\begin{array}{c}\text { Curso } \\
\text { académico }\end{array}$ & $\begin{array}{c}\text { Contestación } \\
\text { en } \mathbf{3} \text { días }\end{array}$ & $\begin{array}{c}\text { Contestación } \\
\text { de } \mathbf{3} \text { a } 5 \text { días }\end{array}$ & $\begin{array}{c}\text { Contestación } \\
\text { de } \mathbf{5} \text { a 10 } \\
\text { días }\end{array}$ & $\begin{array}{c}\text { Contestación } \\
\text { en más de 10 } \\
\text { días }\end{array}$ \\
\hline $\mathbf{2 6}$ & $2013-14$ & $6 \%$ & $23 \%$ & $40 \%$ & $31 \%$ \\
$\mathbf{5 1}$ & $2014-15$ & $35 \%$ & $42 \%$ & $15 \%$ & $8 \%$ \\
$\mathbf{8 5}$ & $2015-16$ & $62 \%$ & $21 \%$ & $12 \%$ & $5 \%$ \\
\hline
\end{tabular}

Tabla 1.- Distribución en el tiempo de entrega de las modificaciones en las fichas, durante tres cursos académicos.

Debemos considerar que la ficha o fichas suministradas al alumno al principio del proceso deben ser suficientemente atractivas y que sean potencialmente significativas para que partiendo de una disposición favorable a aprender más sobre el tema planteado que según Ausubel (2002) implica motivación, comprensión sistematización y transferencia. Que el alumnos sea capaz, tenga o se le genere una predisposición para relacionar este material que se le proporciona y al mismo tiempo conseguir que el tema sea enlazable con conocimientos que ya posea (Rodríguez, 2008), de forma que esté dispuesto a aumentar y mejorar los conocimientos.

\section{Conclusiones}

Con estas técnicas aplicadas pretendemos que al seguir el alumno en concreto y el grupo de alumos un proceso de construcción progresiva de las fichas de los materiales vegetales, estos datos que no se pretende se memoricen, se retienen, al ser significativa su generación, más tiempo y sobre todo se adquiere una forma de actuar una metodología actuacional de amplia aplicación en el sector agronómico y antropológico, para conocer los materiales con los que se trabaja y sobre todo cómo buscar e implementar los requerimientos que en unos 
casos se buscan y en otros se almacenan con fin evidentemente comparativo y base para construir unos razonamientos críticos dentro de las ciencias morfológicas y pomológicas.

Hemos logrado que el alumno sea quien desarrolla su propio conocimiento, y ejercite las competencias, siendo esta la forma de que el alumnado desarrolle progresivamente las competencias a lograr en los futuros egresados.

\section{Referencias}

AUSUBEL, D. P. (2002). Psicología educativa. Un punto de vista cognoscitivo. Pidos Ed. Madrid.

BISCARRI, Félix; LEÓN, Carlos; MOLINA, Francisco Javier Y MOLINA, Alberto (2006). Integración de la simulación informática no presencial y la enseñanza tradicional. Un método de evaluación continúa. Revista Pixel-Bit, 28, 1-7.

CANDOLLE, A.(1813). Théorie élémentaire de la botanique, ou Exposition du principe de la classification naturelle et de l'art de décrire et d'étudier les végétaux. Paris: Déterville.

RODRIGUEZ, M. (2008). La teoría del aprendizaje significativo en la perspectiva de la psicología cognitiva. Octaedro Ed. Barcelona.

RODRIGUEZ, M. (2011). Teoría del aprendizaje significativo: una revisión aplicable a la escuela actual. Rev. Elect. D'investigació i innovació educativa i socioeducativa. 3.1.29-50.

DÍAZ, F. (1999). Estrategias docentes para un aprendizaje significativo. MacGraw-Hill. Interamericanas. Méjico.

LUISEL, V., RODRÍGUEZ, C. (2014). Metodologías de enseñanza para una parendizaje significativo de la histología. RDU. Revista digital universitaria. UNAM. Mexico.

MARCELO, Carlos (2007). Propuesta de Estándares de Calidad para programas de formación docente a través de estrategias de aprendizaje abierto y a distancia. En www.unesco.cl/medios/biblioteca/documentos/estandares_en_e_learning.doc.

NEGROUL, A. M. (1946). Origin and classification of cultured grape. Russ. In: The Ampelography of the USSR, VoL 1,159. Pischepromizdat, Moscow.

VAVILOV N. (1987). Origin and Geography of Cultivated Plants. Cambridge University Press. UK. 